\title{
11. Friedens - und Sicherheitspolitik
}

\section{Christoph Stamm}

\section{(2) OpenEdition}

\section{Journals}

Electronic version

URL: http://journals.openedition.org/sjep/229

DOI: 10.4000/sjep.229

ISSN: 1663-9677

\section{Publisher}

Institut de hautes études internationales et du développement

\section{Printed version}

Date of publication: 1 avril 2004

Number of pages: 201-214

ISSN: $1660-5926$

\section{Electronic reference}

Christoph Stamm, «11. Friedens - und Sicherheitspolitik», Schweizerisches Jahrbuch für

Entwicklungspolitik [Online], 23-1 | 2004, Online erschienen am: 29 April 2010, abgerufen am 08 September 2020. URL : http://journals.openedition.org/sjep/229 ; DOI : https://doi.org/10.4000/sjep. 229

(c) The Graduate Institute 


\section{Friedens- und Sicherheitspolitik*}

D as SCHWEIZER PARLAmENT WAR 2003 aUfGerUfen, zwei Rahmenkredite und ein Bundesgesetz zur zivilen Friedensförderung zu verabschieden. Die Inhalte der Vorlagen waren wenig umstritten. Bei der Höhe des Kredites folgte der Ständerat jedoch nicht dem Vorschlag des Bundesrates, sondern votierte für eine Senkung der Ausgaben.

Einen Schwerpunkt der friedenspolitischen Aktivitäten der Schweiz bildete im Jahr 2003 das Engagement für die Friedenskonsolidierung in Sri Lanka. Diverse Schweizer Institutionen waren auf verschiedenen Ebenen aktiv, um den im Jahr 2001 begonnenen Friedensprozess positiv zu beeinflussen.

Neben der Politischen Abteilung IV des Departements für auswärtige Angelegenheiten (EDA) und dem Verteidigungsdepartement (VBS) sind auch die Direktion für Entwicklung und Zusammenarbeit ${ }^{l}$ (DEZA) sowie Nichtregierungsorganisationen $^{2}$ in der Konfliktbearbeitung tätig. Davon zeugen das Frühwarnprojekt FAST und die Friedensbemühungen in der indonesischen Provinz Aceh.

Das in den neunziger Jahren entstandene und auf das Individuum ausgerichtete Konzept der Menschlichen Sicherheit wurde auch von den Akteuren der schweizerischen Aussenpolitik aufgenommen. Die Politische Abteilung IV des EDA ist unter dem Etikett „Menschliche Sicherheit“ in verschiedenen Bereichen aktiv. Dazu gehören namentlich das „Netzwerk für Menschliche Sicherheit“, die weltweite Eliminierung von Personenminen und die Kontrolle des internationalen Kleinwaffenhandels.

Auch über ein Jahrzehnt nach der Auflösung der Sowjetunion sind die Altlasten des Rüstungsbereichs, welche eine Gefahr für Sicherheit, Gesundheit und Umwelt darstellen, noch nicht beseitigt. Mit einem Kredit hilft die Schweiz bei der Umsetzung des Chemiewaffenübereinkommens und der Vernichtung des russischen Chemiewaffenarsenals.

Im Mai 2003 stimmten Volk und Stände für die Armeereform (Armee XXI). Die Schweizer Armee wird damit an die veränderte sicherheitspolitische Lage angepasst. Die Sicherheit der Schweiz soll künftig durch eine vertiefte internationale Kooperation gewährleistet werden.

* Von Christoph Stamm, Politologe.

1 Die DEZA hat im Jahr 2003 Leitlinien zur Friedensentwicklung vorgelegt. Bereits in der Strategie 2010 hatte die DEZA die Krisenprävention und -bewältigung zu einem ihrer fünf thematischen Schwerpunkte erklärt. Siehe auch Jahrbuch 2004, Nr. 1, Kap. 2, „Zusammenarbeit mit den Entwicklungsländern“.

2 Diese können zum Teil auf massgebliche politische und finanzielle Unterstützung des Bundes zählen. Für eine Übersicht siehe: Ribaux, Claude André, Friedensförderung und Konfliktbearbeitung in der Schweiz. Eine Bestandesaufnahme. Typotron, St. Gallen, 2003. 


\subsection{Gesetzesvorlagen und Rahmenkredite mit friedenspolitischen Inhalten}

$\square$ Bundesgesetz und Rahmenkredit zur zivilen Friedensförderung und zur Stärkung der Menschenrechte

Der vom Bundesrat im Jahr 2002 vorgelegte neue Gesetzesentwurf über Massnahmen zur zivilen Friedensförderung und Stärkung der Menschenrechte war in beiden Räten wenig umstritten ${ }^{3}$. Das Bundesgesetz wurde im März 2003 vom Nationalrat mit 107 zu 24 Stimmen und im September desselben Jahres vom Ständerat mit kleinen Modifizierungen einstimmig angenommen ${ }^{4}$.

Der Nationalrat stimmte auch dem entsprechenden vierjährigen Rahmenkredit über 240 Millionen Franken mit 120 zu 27 Stimmen deutlich zu. Mehr zu reden gab der Rahmenkredit im Ständerat. Bundesrätin Calmy-Rey plädierte vergebens für die geplanten 240 Millionen Franken. Der Bundesrat habe die zivile Friedensförderung als strategische Priorität festgelegt, und die Mehrausgaben seien anderswo in ihrem Departement (EDA) eingespart worden. Der Rat entschied sich mit $27 \mathrm{zu} 10$ Stimmen gegen den Bundesrat und für eine Kürzung des Rahmenkredites auf 200 Millionen Franken (24 gegen 3 Stimmen). Im Dezember 2003 trafen sich die beiden Räte schliesslich in der Mitte und einigten sich auf einen Kredit über 220 Millionen Franken.

Da Jahrbuch 2003, Nr. 1, Kap. 1.2., „Bundesgesetz zur zivilen Friedensförderung und zur Stärkung der Menschenrechte", S. 7-8.

\section{$\square$ Rahmenkredit für zivile friedensfördernde Massnahmen im Rahmen des VBS}

Im Dezember 2002 legte der Bundesrat eine Botschaft über einen Rahmenkredit für zivile friedensfördernde Massnahmen im Rahmen des Verteidigungsdepartementes (VBS) vor ${ }^{5}$. Der Kredit über 180 Millionen Franken für den Zeitraum 2004-2007 soll zur Finanzierung der zivilen friedensfördernden Aktivitäten des VBS dienen. Es handelt sich dabei hauptsächlich um das Genfer Zentrum für Sicherheitspolitik, das Genfer Internationale Zentrum für Humanitäre Minenräumung, das Genfer Zentrum für die Demokratische Kontrolle der Streitkräfte und das International Relations and Security Network der ETH Zürich (mit jeweils $15 \%, 18 \%, 23 \%$ und 19\% des Gesamtbudgets) ${ }^{6}$. Daneben werden verschiedene Aktivitäten im Rahmen der NATO-Partnerschaft für den Frieden sowie bilaterale oder multilaterale Kooperations- und Unterstützungsprogramme finanziert. In den nächsten vier Jahren sollen die bestehenden Aktivitäten fortgeführt und konsolidiert werden.

Der Bund möchte mit einem als „Haus des Friedens“ (,Maison de la Paix“) benannten Bau bis 2007 alle Genfer Zentren örtlich vereinigen und weiteren

3 Bundesrat, Rahmenkredit und Bundesgesetz über Massnahmen zur zivilen Konfliktbearbeitung und Menschenrechtsförderung (02.076, 02.077), Bern, 23. Oktober 2002.

4 Der Ständerat strich die Bildung einer Expertenkommission aus dem Gesetz und ermöglichte dem Bund die Unterstützung wissenschaftlicher Institutionen. Mit einem Postulat wurde der Bundesrat aufgefordert, die politische Führung und Koordination der zivilen Friedensförderung und Konfliktbearbeitung des Bundes departementsübergreifend zu strukturieren. Dem Parlament ist entsprechend Bericht zu erstatten (Postulat 03.3178).

5 Bundesrat, Botschaft über einen Rahmenkredit für zivile friedensfördernde Massnahmen im Rahmen des VBS (02.091), Bern, 9. Dezember 2002 (BB1 2003 622).

6 Für eine Beschreibung der Zentren siehe Jahrbuch 2003, S. 211-213. 
Institutionen, welche in verwandten Bereichen tätig sind, Räumlichkeiten zur Verfügung stellen. Mit diesem Projekt sollen laut Bundesrat die Ausstrahlungskraft der Institutionen weiter erhöht, die Effizienz gefördert, die Kontakte und Zusammenarbeit mit anderen Institutionen in Genf intensiviert und das internationale Genf in den Bereichen Sicherheitspolitik und Frieden gestärkt werden. Die für den Bau des Projekts „Maison de la Paix“ erforderlichen Mittel waren nicht Gegenstand des Antrages; sie werden mit einer zukünftigen Immobilienbotschaft beantragt werden.

Der Rahmenkredit war in beiden Parlamentskammern unumstritten. Er wurde im März 2003 vom Nationalrat mit 122 zu 10 Stimmen und im September 2003 vom Ständerat mit kleinen Modifizierungen einstimmig angenommen.

\subsection{Wichtige friedenspolitische Aktivitäten}

\section{$\square$ Beschwerliche Friedenskonsolidierung in Sri Lanka}

Unter der Vermittlung Norwegens und dem Druck der USA und Indiens führte ein im Februar 2002 geschlossener, unbefristeter Waffenstillstand zwischen der Regierung und den tamilischen Befreiungstigern (LTTE) zu einer Deeskalation des seit 1983 andauernden Bürgerkriegs in Sri Lanka. Bis heute hat der Konflikt etwa 70'000 Menschenleben gefordert und 1,5 Millionen Menschen zu Flüchtlingen gemacht.

Nach mehreren Verhandlungsrunden zwischen Regierung und LTTE geriet der Friedensprozess im Frühjahr 2003 ins Stocken, bevor der grosse politische Durchbruch erzielt worden wäre. Die Menschenrechtslage hat sich zwar etwas verbessert ${ }^{7}$, doch die immer noch herrschende Unsicherheit erschwert den Wiederaufbau - die Friedensdividende lässt auf sich warten ${ }^{8}$.

Da die Schweiz in den letzten 20 Jahren mehr als 35'000 tamilische Flüchtlinge aufgenommen hat, misst die eidgenössische Aussenpolitik Sri Lanka eine besondere Bedeutung zu. Folgende Beispiele zeigen, wie mehrere Schweizer Institutionen den Friedensprozess mit verschiedenen Projekten unterstützen.

- Das schweizerische Aussenministerium (EDA) finanziert zusammen mit Deutschland das Resource Network for Conflict Studies and Transformation Sri Lanka (RNCST) des Berghof-Zentrums für Konfliktforschung in Berlin. Die Arbeit des RNCST zielt darauf ab, die Kapazitäten für eine konstruktive Konflikttransformation in Sri Lanka auf allen „Tracks“9 zu stärken und Foren sowie Strukturen für Dialog und Verständigung zu schaffen. Durch verschiedene Massnahmen soll eine Ergänzung und Stärkung der bereits vorhandenen, einheimischen Potenziale in den Bereichen Ausbildung, Forschung und Dialog erreicht werden ${ }^{10}$.

Amnesty International, Jahresbericht 2003, Fischer, Frankfurt, 2003.

8 Die im Juni 2003 von der internationalen Gebergemeinschaft gesprochenen 4,5 Milliarden US-Dollar können noch nicht abgerufen werden. Wochenzeitung, 11. September 2003.

9 Mit „Track I bis III“ werden verschiedene komplementäre Vermittlungsansätze in der Konfliktbearbeitung bezeichnet.

10 Berghof Forschungszentrum für konstruktive Konfliktbearbeitung: <www.berghof-center.org $>$. Berghof Foundation for Conflict Studies : <www.berghof-foundation.lk>. 
ـ Die Konfliktparteien haben sich Ende 2002 darauf geeinigt, eine politische Lösung des ethnopolitischen Konflikts im Rahmen föderaler Strukturen zu suchen. Die bisherige stark zentralistische Funktionsweise des Staates soll überwunden werden. Die Schweiz unterstützt die Partner in Sri Lanka mittels Erfahrungsaustausch zu Themen wie Konstitutionalismus, Föderalismus, dezentrale Strukturen und Ziviladministration. Das EDA und das Freiburger Institut für Föderalismus führen in diesem Bereich Beratungen und Schulungen durch. Im Jahr 2003 besuchten mehrere Delegationen mit sri-lankischen Ministern, Parlamentariern, Vertretern der LTTE sowie Journalisten die Schweiz, um das schweizerische föderale System besser kennen zu lernen ${ }^{11}$.

$\checkmark$ Mit finanzieller Unterstützung des EDA erstellte die Schweizerische Stiftung für Minenräumung (FSD) für das Entwicklungsprogramm der Vereinten Nationen (UNDP) eine umfassende Übersicht über minenverseuchte Gebiete, bildete nationale Hilfskräfte aus und stellte kleine Notfallkräfte für Räumungsoperationen zur Verfügung ${ }^{12}$.

๑ Ein von Helvetas geleitetes Projekt hat Versöhnung, Frieden und Entwicklung im Nordosten Sri Lankas zum Ziel ${ }^{13}$. Bei der ins Projekt integrierten Friedensförderung stehen die Konfliktminderung und die Förderung des gegenseitigen Verständnisses der Bevölkerungsgruppen durch praktische Zusammenarbeit sowie kulturelle und sportliche Treffen im Vordergrund. Mit dem Aufbau von lokalen Netzwerken friedensorientierter NRO soll bei den Entscheidungsträgern für gewaltfreie Lösungen lobbyiert werden.

- Mit periodischen Gesprächen am runden Tisch ermöglicht das Kompetenzzentrum Friedensförderung (KOFF) Vertretern von Schweizer Regierungsstellen und Nichtregierungsorganisationen, sich über aktuelle Trends und Herausforderungen im Bereich der Friedensförderung in Sri Lanka auszutauschen ${ }^{14}$.

\section{$\square$ Gescheiterte Friedensbemühungen in der indonesischen Provinz Aceh}

Seit 1976 führt die Bewegung Freies Aceh (GAM) einen bewaffneten Kampf um die Unabhängigkeit der indonesischen Provinz Aceh. Die nördlichste Provinz Indonesiens ist reich an Rohstoffen, von deren Ausbeutung die lokale Bevölkerung kaum profitiert. Ermutigt wurden die Separatisten durch die Loslösung Osttimors von Indonesien und die Gründung des Staates Timor-Leste im Jahr 2002. Die indonesische Regierung und das Militär wollen eine Abspaltung Acehs mit allen Mitteln verhindern.

Vor diesem Hintergrund bemühen sich zwei in der Schweiz ansässige NRO um Frieden und die Achtung der Menschenrechte. Es handelt sich dabei um die Genfer Institution Centre for Humanitarian Dialogue ${ }^{15}$ (HDC) und die transnationale Organisation Peace Brigades International ${ }^{16}$ (PBI), deren Schweizer Büro in Freiburg ansässig ist. Die unterschiedlichen friedenspolitischen Ansätze der beiden NRO können als komplementär eingestuft werden.

11 Das EDA möchte die positiven Erfahrungen der Schweiz mit dem Föderalismus als komparativen Vorteil im Bereich der Friedensförderung vermehrt nutzen.

12 Schweizerische Stiftung für Minenräumung, Jahresbericht $2002:<w w w . m i n e a c t i o n . c h>$.

13 Helvetas: <www.helvetas.ch>.

14 KOFF Newsletter 07/2003, 1. September 2003.

15 Center for Humanitarian Dialogue: <www.hdcentre.org $>$.

16 Peace Brigades International: <www.peacebrigades.org $>$. 
Die Arbeit des Centre for Humanitarian Dialogue konzentriert sich auf die Förderung humanitärer Prinzipien, die Prävention von Konflikten und die Milderung der Konsequenzen von Konflikten durch Dialog. Seine Tätigkeit wurde vom Bund 2003 mit einer Million Franken unterstützt.

Im Jahr 2000 begann das Zentrum den Dialog zwischen der indonesischen Regierung und der GAM zu fördern. Die Konfliktparteien trafen sich ab 2001 unter der Leitung der Genfer Institution zu Gesprächen und zeigten sich zunehmend bereit, Mechanismen zur Konfliktlösung zu etablieren. Nach verschiedenen vertrauensbildenden Massnahmen und weiteren Verhandlungsrunden unterzeichneten die Rebellen mit der indonesischen Regierung Ende 2002 in Genf ein Waffenstillstandsabkommen, welches Aceh Autonomie zugesteht. Im Januar 2003 starteten die aus Überwachern des HDC, der indonesischen Regierung und der GAM bestehenden tripartiten Gruppen mit der Monitoring-Operation zur Überprüfung des Waffenstillstandes. Verschiedene Friedenszonen konnten eingerichtet werden, und interne Flüchtlinge konnten in ihre Häuser zurückkehren. Das HDC konnte jedoch nicht verhindern, dass der Friedensprozess im Verlauf des Frühjahrs 2003 ins Stocken geriet. Die Bewegung Freies Aceh und das indonesische Militär hielten sich nicht an den vereinbarten Friedensplan und zeigten sich kompromisslos. Die Verhängung des Kriegsrechts im Mai 2003 und der Beginn neuer Kampfhandlungen beendeten den Friedensprozess und erzwangen den Rückzug des Zentrums für Humanitären Dialog. Dessen wichtigstes Projekt ist damit vorläufig gescheitert.

Die Internationalen Friedensbrigaden (PBI) setzen sich in Krisen- und Konfliktgebieten für den Schutz der Menschenrechte und die gewaltfreie Konfliktbearbeitung ein. PBI unterstützt mit Freiwilligen-Teams durch die Schaffung von Freiräumen jene zivilgesellschaftlichen Organisationen, welche sich mit gewaltfreien Methoden für Menschenrechte, soziale Gerechtigkeit und die friedliche Bearbeitung von Konflikten einsetzen. PBI-Schweiz gehört zu den bedeutendsten Ländersektionen und erhält von schweizerischen öffentlichen Institutionen finanzielle Unterstützung. Letztere bestritten in den Jahren 2001/2002 38 Prozent des Budgets. PBI-Schweiz lieferte ihrerseits 45 Prozent ihrer Ressourcen an Peace Brigades International mit Sitz in London ab.

PBI wurde im Jahr 2000 auf Anfrage von indonesischen Menschenrechtsgruppen aktiv und öffnete Büros in Jakarta, Banda Aceh und Lhokseumawe (beide Provinz Aceh). Im Jahr 2003 bestand das Indonesienprojekt aus 20 Freiwilligen. Diese begleiteten gefährdete Personen verschiedener Organisationen, um einen Freiraum zu schaffen, der es den Gruppen ermöglicht, ihre Arbeit trotz des Klimas von Gewalt und Angst fortzuführen. Das Projektbüro in Jakarta hält zudem den Kontakt zu Regierungsstellen, Auslandvertretungen, nationalen wie internationalen Organisationen und beobachtet die politische Entwicklung. Nach Wiederausbruch des bewaffneten Konfliktes in Aceh wurde PBI im Juni 2003 von der indonesischen Regierung gezwungen, ihre Büros in der Provinz zu schliessen und die Region zu verlassen. 


\section{$\square$ Das Frühwarnsystem FAST}

Das Frühwarnsystem für Konflikte Early Recognition of Tension and Fact-Finding (FAST) wurde 1998 im Auftrag der DEZA gebildet ${ }^{17}$. Das Projekt wird unter der Leitung der Schweizerischen Friedensstiftung Swisspeace geführt. Ziel von FAST ist es, politische Entwicklungen und Eskalationen zu identifizieren, bevor diese zu einem bewaffneten Konflikt führen. Dadurch sollen politische Entscheidungsträger in der Lage sein, kohärente Strategien zur Verhinderung oder Limitierung destruktiver Entwicklungen zu formulieren und Möglichkeiten zur Konfliktprävention zu identifizieren. FAST ist auf die geografischen Bedürfnisse der schweizerischen Aussenpolitik ausgerichtet, und die Anzahl der analysierten Länder wird kontinuierlich erhöht. Das Projekt analysierte im Jahr 2003 24 Länder oder Gebiete in folgenden Grossregionen: Region der grossen Seen in Afrika, Horn von Afrika, südliches Afrika, Zentralasien, Kaukasus und Balkan. Das Projekt unterscheidet sich von anderen Frühwarnsystemen durch zwei Merkmale. Erstens kombiniert es quantitative und qualitative Methoden. Die quantitativen Informationen stammen aus der Analyse von Ereignisdaten und aus den Informationen lokaler Netzwerke. Die qualitativen Analysen werden von Länderexperten geliefert. Zweitens ist FAST in den politischen Entscheidungsfindungsprozess eingebunden und füllt damit die Lücke zwischen Frühwarnung (early warning) und frühem Handeln (early action).

Arbeiten sind im Gang, um neue Prognosemodelle über das konfliktive und kooperative Geschehen in den Zielländern zu entwickeln. Neben ausgebauten wissenschaftlichen Partnerschaften soll das Projekt mit einer internationalen Trägerschaft in Richtung „FAST International“" weiterentwickelt werden. Im Jahr 2003 wurde diesbezüglich mit ausländischen Entwicklungsagenturen intensiv verhandelt.

Eine externe Evaluation der Schweizerischen Friedensstiftung kam zum Schluss, dass FAST in ihrem Bereich internationale Pionierarbeit geleistet hat ${ }^{18}$. Bedauert wurde hingegen die mangelnde Präsenz von FAST in der internationalen friedenswissenschaftlichen Gemeinde ${ }^{19}$. Gründe dafür seien der Zeitmangel der Projektwissenschaftler und die Vertraulichkeitsvorschriften des Auftraggebers DEZA.

\subsection{Menschliche Sicherheit}

\section{$\square$ Menschliche Sicherheit - Erläuterungen zu einem Konzept}

Nachdem sich Sicherheitspolitik in der Vergangenheit auf die Sicherheit von Staaten beziehungsweise zwischen Staaten sowie auf die globale Sicherheit (atomare Sicherheit) konzentrierte, ist seit den neunziger Jahren die Sicherheit auf der individuellen Ebene verstärkt ins Blickfeld von Wissenschaft und Politik gerückt. Die Hauptgründe für diesen Wandel sind erstens die Verlagerung von

\footnotetext{
Frühwarnsystem FAST : <www.swisspeace.org/fast>.

18 Evaluation der Schweizerischen Friedensstiftung „Swisspeace“, durchgeführt vom Zentrum für Wissenschafts- und Technologiestudien (CEST) im Auftrag des Bundesamtes für Bildung und Wissenschaft, <www.cest.ch>.

19 „Hier besitzt die Schweiz ein wissenschaftliches Produkt von absoluter Weltspitzenklasse, und viel zu wenige wissen es." Harald Müller in ebd.
} 
Gewaltkonflikten vom zwischenstaatlichen in den innerstaatlichen Bereich und die damit einhergehende massive Zunahme von zivilen Opfern. Es begann sich die Überzeugung durchzusetzen, dass dauerhafte Stabilität und die Vermeidung von gewalttätigen Konflikten nicht ohne die Gewährleistung eines Minimums an individueller Sicherheit erreicht werden können. Zweitens sind im Bereich der Konfliktprävention langfristige Massnahmen ins Blickfeld getreten, welche sich nicht mehr nur auf diplomatische und militärische Aktivitäten beschränkten, sondern verstärkt wirtschaftliche, soziale und politische Stossrichtungen verfolgten. Individuelle wirtschaftliche und soziale Entwicklungen als Teile einer umfassenden Strategie zur Prävention gewaltsamer Konflikte erhielten damit auch aus sicherheitspolitischer Perspektive einen Bedeutungszuwachs. Parallel dazu wuchs drittens im Entwicklungssektor das Verständnis für die Sicherheitsrelevanz der Entwicklungszusammenarbeit. Ein auf individueller Ebene sicheres und stabiles Umfeld wird als unverzichtbare Rahmenbedingung für eine erfolgreiche wirtschaftliche, soziale und politische Entwicklung in einem Land angesehen.

Gemeinsamer Nenner dieser jeweiligen Veränderung der Standpunkte im Sicherheits- und Entwicklungssektor ist der Konsens, dass es ohne Entwicklung keine Sicherheit, und ohne Sicherheit keine Entwicklung geben wird. Menschliche Sicherheit (human security) bildet damit einen Brennpunkt gemeinsamer Anliegen ${ }^{20}$.

Das Konzept der Menschlichen Sicherheit wurde im Human Development Report 1994 erstmals breit lanciert ${ }^{21}$. Laut Bericht gelten folgende sieben Kategorien als potenzielle Gefahrenbereiche, in welchen Menschliche Sicherheit erreicht werden soll: wirtschaftliche Sicherheit, Nahrungssicherheit, gesundheitliche Sicherheit, Umweltsicherheit, persönliche Sicherheit, Sicherheit der Gemeinschaft sowie politische Sicherheit. Auf einen einfachen Nenner gebracht, forderte das UNDP-Konzept der Menschlichen Sicherheit Freiheit von Furcht und Freiheit von Not (freedom from fear, freedom from want) für alle Individuen. Der Vorschlag im Entwicklungsbericht diente als Ausgangspunkt für zahlreiche Publikationen, die sich mit dem Konzept kritisch auseinandersetzten und ihrerseits Entwürfe und Definitionen zum Thema einbrachten. In der Praxis wird heute zwischen einer engen Auslegung der Menschlichen Sicherheit unterschieden, welche sich auf Gewaltaspekte konzentriert (freedom from fear), und einer breiten Auslegung, welche Aspekte der menschlichen Entwicklung miteinbezieht. Beiden Ansätzen gemeinsam ist die Orientierung am Individuum und seinem Bedürfnis nach Sicherheit.

Das Human Security Center der University of British Columbia möchte mit der Erarbeitung eines jährlichen Human Security Reports die Menschliche Sicherheit und deren Entwicklung messbar machen ${ }^{22}$. Der Bericht, der sich auf den Gewaltaspekt der menschlichen Sicherheit konzentriert, soll den Human Development Report des UNDP ergänzen.

20 Trachsler Daniel, „Menschliche Sicherheit: Konzept und Praxis“, in Wenger Andreas (Hg.), Bulletin 2003 zur schweizerischen Sicherheitspolitik, Forschungsstelle für Sicherheitspolitik der ETHZ, Zürich, 2003.

21 Human Development Report 1994, UNDP, New York, 1994.

22 Human Security Center, University of British Columbia: <www.ligi.ubc.ca/chs.htm>, Human Security Report: <www.humansecurityreport.info>. Dieser Bericht wird unter anderem vom Netzwerk für 


\section{$\square$ Die Menschliche Sicherheit in der schweizerischen Aussenpolitik}

Neben anderen Staaten ${ }^{23}$ hat auch die Schweiz das Konzept der Menschlichen Sicherheit aufgegriffen. Zu einer einheitlichen Definition ist es aber bisher noch nicht gekommen, was Kommentatoren denn auch kritisieren ${ }^{24}$. Im Aussenpolitischen Bericht 2000 wird mit dem Hinweis auf den Millennium Report von UNO-Generalsekretär Kofi Annan das Konzept der Globalen Menschlichen Sicherheit vorgestellt: „Dieses Konzept umfasst nicht nur die Freiheit von Furcht (Sicherheitsagenda), sondern auch die Freiheit von Not (Entwicklungsagenda) sowie eine nachhaltige Zukunft (Umweltagenda). Die Verbesserung der Menschlichen Sicherheit ist nur möglich, wenn die Grundsätze der Guten Regierungsführung durchgesetzt werden" ${ }^{25}$. Der Begriff wird an weiteren Stellen verwendet, aber nie klar definiert oder konkretisiert.

Verwendet die Direktion für Entwicklung und Zusammenarbeit das Konzept, meint sie die breite Definition der Menschlichen Sicherheit: „Sicherheit ist umfassend zu verstehen - es geht um das Wohlergehen des einzelnen Menschen. ... Die Basis jeder wirksamen Sicherheitspolitik liegt deshalb in der Armutsbekämpfung“ “26. Es stellt sich damit die Frage, ob mit dieser breiten Definition jegliche Entwicklungszusammenarbeit, welche sich direkt an die einzelnen Menschen richtet, in den Bereich der Menschlichen Sicherheit fällt.

Spricht dagegen die Politische Abteilung IV des EDA von Menschlicher Sicherheit, so denkt sie an Freiheit von Gewalt: „Wie die meisten nördlichen Staaten konzentriert sich auch die Schweiz auf Gewaltaspekte der Menschlichen Sicherheit." Relativ früh erkannte das EDA den nützlichen Handlungsansatz des Konzeptes $^{27}$. Zu den ersten Aktivitäten, in welchen sich die Schweiz unter dem Etikett der Menschlichen Sicherheit profilierte, gehörte der Kampf gegen Personenminen, der mit dem Übereinkommen über das Verbot von Personenminen (Ottawa-Konvention von 1997) international zum Erfolg führte. Im Jahr 2001 wurde die PA IV und die PA IIIb zur „Politischen Abteilung IV, Menschliche Sicherheit (Frieden, Menschenrechte, Humanitäre Politik)“ zusammengeschlossen. Die Namensgebung ist ein Zeichen dafür, dass das EDA dem Konzept grosse Bedeutung zumisst. Unter dem Oberbegriff der Menschlichen Sicherheit ist die Schweiz heute in folgenden Bereichen aktiv: Kampf gegen die Kleinwaffenproliferation, Unterstützung des Internationalen Strafgerichtshofs, Smart Sanctions, Kinder in Konfliktsituationen, Einbindung bewaffneter nichtstaatlicher Akteure, humanitäres Völkerrecht, Auswirkungen von multinationalen Unternehmen in bewaffneten Konflikten und Einhaltung von Verhaltensko-

Menschliche Sicherheit und von der Schweiz unterstützt; seine erste Ausgabe war bei Redaktionsschluss noch nicht erschienen.

23 Eine Vorreiterrolle spielten Kanada und Japan. Siehe auch <www.humansecurity.gc.ca>.

24 Siehe Tanner Fred, „Menschliche Sicherheit - neue Diplomatie“, Neue Zürcher Zeitung, 13. Januar 2003 sowie Trachsler Daniel, „Menschliche Sicherheit: Konzept und Praxis“, in Wenger Andreas (Hg.), Bulletin 2003 zur schweizerischen Sicherheitspolitik, Forschungsstelle für Sicherheitspolitik der ETHZ, Zürich, 2003.

25 Millennium Report of the Secretary-General of the United Nations, <www.un.org/millennium/sg/ report/>; Bundesrat, Aussenpolitischer Bericht 2000 - Präsenz und Kooperation: Interessenwahrung in einer zusammenwachsenden Welt, Bern, 2000.

26 DEZA, Jahresthema 2003 „Sicherheit durch Entwicklung“, <www.deza.ch>.

27 Maurer Peter, „Die Zivilbevölkerung besser schützen“, Neue Zürcher Zeitung, 2. Dezember 2002. 
dizes $^{28}$. Zudem ist die Schweiz ein aktives Mitglied des Netzwerks Menschliche Sicherheit (Human Security Network), initiierte ein News Bulletin und unterstützt Forschungsprojekte zum Thema Menschliche Sicherheit ${ }^{29}$.

\section{$\square$ Netzwerk für Menschliche Sicherheit}

Die Aussenminister des überregionalen Netzwerks für Menschliche Sicherheit trafen sich im Mai 2003 in Österreich zum fünften Ministertreffen ${ }^{30}$. Im Zentrum der Diskussionen standen die Themen „Menschenrechtserziehung“ und „Kinder in bewaffneten Konflikten.“ Es wurden politische Strategien weiterentwickelt, wie die Erziehung zur besseren Einhaltung der Menschenrechte und damit zur Menschlichen Sicherheit beitragen kann, wie Kinder vor den Auswirkungen von gewalttätigen Konflikten geschützt werden können und wie das Netzwerk in multilateralen Gremien verstärkt aktiv werden kann ${ }^{31}$. Die Schweiz möchte innerhalb des Netzwerks unter anderem die Themen „Stärkung des humanitären Völkerrechts“ und „Rolle multinationaler Unternehmen in Konfliktsituationen"vertiefen ${ }^{32}$.

\section{$\square$ Kleinwaffenkontrolle}

An der im Juli 2003 in New York tagenden Internationalen Konferenz zur Kleinwaffenkontrolle wurde eine Zwischenbilanz der Umsetzung des zwei Jahre alten, nicht rechtsverbindlichen UN-Aktionsprogramms zur Kleinwaffenkontrolle gezogen ${ }^{33}$. Rund hundert Staaten berichteten über nationale Programme zur Eindämmung der illegalen Verbreitung und des Missbrauchs von Kleinwaffen.

Jährlich fordert der Gebrauch von Kleinwaffen eine halbe Million Menschenleben. Deshalb ist die Kleinwaffenkontrolle für viele Länder zu einem ernsthaften Thema der Menschlichen Sicherheit geworden. Dieser erhöhten Sensibilität stehen aber bedeutende Wirtschaftsaktivitäten gegenüber. Der Wert der jährlich produzierten Kleinwaffen beträgt 7,4 Milliarden US-Dollar, und in vielen Ländern ist die Waffenlobby sehr stark $^{34}$. Nur wenn das UN-Aktionsprogramm durch verbindliche Regeln ergänzt wird, können Vereinbarungen zur Exportkontrolle und Markierung von Waffen Wirkung zeigen ${ }^{35}$.

28 Siehe Bundesrat, Botschaft über einen Rahmenkredit für Massnahmen zur zivilen Konfliktbearbeitung und Menschenrechtsförderung (02.076), Bern, 2002 (BB1 2002 7975).

29 Human Security Network News Bulletin: <www.hsph.harvard.edu/hpcr/human_security.htm>; z. B. die Publikation Putting People First: Human Security Perspectives on Small Arms Availability and Misuse, Centre for Humanitarian Dialogue, Juli 2003, <www.hdcentre.org/Programmes/small.htm>.

30 Die Mitglieder sind: Kanada, Norwegen, Österreich, Slowenien, Griechenland, Irland, die Niederlande, Chile, Thailand, Jordanien, Mali, die Schweiz und Südafrika (als Beobachter). Netzwerk für Menschliche Sicherheit: <www.humansecuritynetwork.org>.

31 Folgende Initiativen wurden verabschiedet: Grazer Deklaration über die Prinzipien der Menschenrechtserziehung und menschlichen Sicherheit; das Handbuch über Menschenrechtserziehung; die Politikstrategie Bridging the Gap für Kinder in bewaffneten Konflikten sowie ein Kinderrechtstrainingsprogramm für Monitoring und Rehabilitation im Feldeinsatz.

32 Pressemitteilung EDA, 7. Mai 2003.

33 First Biennal Meeting of States on the Implementation of the UN Programme of Action on Small Arms and Light Weapons: <http://disarmament.un.org:8080/cab/salw-2003.html>.

34 The Economist, „One victim every minute“, July $23^{\text {rd }} 2003$.

35 SPICE-Newsletter 4/2003 <www.gtz.de/spice>. 
Die Schweiz hat in Zusammenarbeit mit Frankreich ein Projekt zur Markierung und Rückverfolgung von Kleinwaffen initiiert. Dies soll einen besseren Überblick über die Waffenbestände und -flüsse ermöglichen und Grundlagen für die Unterbindung des illegalen Handels schaffen. Die beiden Länder erarbeiteten eine Machbarkeitsstudie und organisierten Seminare zum Thema. Eine von der UNO eingesetzte Expertengruppe, welcher auch die Schweiz angehört, kam im Juli 2003 zum Schluss, dass die Entwicklung eines internationalen Instruments zur Markierung und Rückverfolgung von Kleinwaffen möglich und wünschenswert ist. Zum Thema Kleinwaffen finanzierte die Schweiz ausserdem Studien des Instituts für Abrüstungsforschung (UNIDIR) und des Genfer Forscherteams Small Arms Survey ${ }^{36}$, welches das Jahrbuch Small Arms Survey 2003 publizierte. Den Schwerpunkt der Ausgabe bildet die Studie über die Konsequenzen der Kleinwaffen auf die menschliche Entwicklung. Die Autoren kommen zum Schluss, dass die Verfügbarkeit und der Missbrauch von Kleinwaffen die menschliche Entwicklung verhindern und Entwicklungsanstrengungen von Gebern, NRO und betroffenen Staaten behindern.

Die internationalen Bemühungen der Schweiz stehen im Kontrast zur Innenpoli$\mathrm{tik}^{37}$. Die Schweiz hat im internen Vergleich eine hohe Kleinwaffendichte und eine sehr liberale Waffengesetzgebung. Auch nach der Armeereform müssen die dienstleistenden Bürger das Sturmgewehr zu Hause aufbewahren und bekommen am Ende der Dienstzeit ihre Ordonnanzwaffe geschenkt. Die Schweizerische Kampagne gegen Kleinwaffen, welche sich unter anderem für die Abschaffung des Rechts auf Waffenbesitz einsetzt, forderte im Juli 2003 Verteidigungsminister Schmid auf, die überzähligen Waffen der Armee nicht weiterzuverkaufen, sondern zu verschrotten ${ }^{38}$.

Mit einer sich in der Vernehmlassung befindenden Teilrevision des Waffengesetzes von 1999 sollen bestehende Lücken geschlossen werden ${ }^{39}$. Das revidierte Gesetz sieht unter anderem die Kontrolle des Waffenhandels, das Verbot von Seriefeuerwaffen und die Registrierung aller privaten Feuerwaffen in einem zentralen Register vor.

Im Oktober 2003 starteten Amnesty International, Oxfam und das Internationale Aktionswerk zu Kleinwaffen (IANSA) in über 60 Ländern eine Kampagne gegen den unkontrollierten Waffenhandel ${ }^{40}$. Obwohl die unkontrollierte Verbreitung und der Missbrauch von Waffen Ursachen vieler Menschenrechtsverletzungen seien, so die Initianten, werde der internationale Waffenhandel kaum kontrolliert. Ziel der Kampagne ist ein rechtlich verbindliches internationales Abkommen, welches alle Rüstungsgütertransfers strikt kontrolliert sowie völker- und menschenrechtsverletzende Waffengeschäfte verbietet. Auch auf nationaler Ebene sollen die Regierungen ihrer Verantwortung gerecht werden, die Waffentransfers stärker kontrollieren und die Bürger besser vor bewaffneter

36 Small arms survey: <www.smallarmssurvey.org $>$.

37 „Die Schweiz kann in diesem Bereich (internationale Kontrolle von Kleinwaffen) nur dann glaubwürdig argumentieren, wenn sie selber eklatante Lücken in der eigenen Waffengesetzgebung geschlossen hat." Neue Zürcher Zeitung, 27. September 2003.

38 Schweizerischer Friedensrat, Kampagne gegen Kleinwaffen: <www.friedensrat.ch/kleinwaffen. hauptseite.html>.

39 Pressemitteilung EJPD, 23. September 2002, 22. September 2003.

40 „Waffen unter Kontrolle“, <www.oxfam.de/article.asp?id=361>. 
Gewalt schützen. In der Schweiz unterstützt Amnesty International die Vorschläge des Bundesrates zur Registrierung aller Waffenkäufe und -verkäufe ${ }^{41}$.

\section{$\square$ Antipersonenminen}

Im September 2003 tagte die fünfte Vertragsparteienkonferenz der Ottawa-Konvention für das Verbot von Antipersonenminen. Die Konferenz bot die Gelegenheit, den Stand der Umsetzung der Konvention zu überprüfen und über das weitere Vorgehen $\mathrm{zu}$ beraten $^{42}$. Die Vertragsstaaten bekundeten den Willen, ihre Verpflichtungen umzusetzen und sich für die Universalisierung der Konvention einzusetzen. Die Teilnehmer wollten jedoch keine zusätzlichen Finanzmittel für Kampagnen gegen Minen und für Minenopfer sprechen. Auf Vorschlag der Schweizer Delegation wurde eine informelle Arbeitsgruppe zur Problematik des Minengebrauches von bewaffneten nichtstaatlichen Akteuren eingesetzt. Diese können mit der Ottawa-Konvention nicht in die Pflicht genommen werden. Seit dem Jahr 2000 verhandelt die NRO Geneva Call mit Rebellengruppen über einen Verzicht von Antipersonenminen. Bislang haben 22 Gruppen die Verpflichtungserklärung von Geneva Call unterzeichnet.

Die Schweiz wendete im Jahr 2002 über 14 Millionen Franken für den Kampf gegen Antipersonenminen auf ${ }^{43}$. Knapp die Hälfte dieses Betrags ging ans Genfer Internationale Zentrum für humanitäre Minenräumung (GICHD). Zu den Schwerpunktländern schweizerischer Entminungsaktivitäten zählten Mosambik, Eritrea, Albanien, Angola und der Tschad. Im Jahr 2002 weitete der Bund die Aktionen auf die Länder Afghanistan, Angola, Kolumbien, Äthiopien, Libanon und Sri Lanka aus. Die grösste in der Minenräumung tätige Schweizer NRO, die Schweizerische Stiftung für Minenräumung, wendete im Jahr 2002 knapp 4 Millionen Franken auf.

Um die Schweizer Hilfsorganisation Menschen gegen Minen (MgM) war im Juni 2003 ein Streit entbrannt. Die deutsche Stiftung (und Mutterorganisation) MgM warf dem Verein MgM Schweiz vor, von den in den Jahren 2000 bis 2002 gesammelten 6,18 Millionen Franken nur 80'000 Franken in Minenräumungsprojekte investiert zu haben. MgM Schweiz stellte dies nicht in Abrede und begründete die Tatsache mit den hohen Aufbaukosten der Organisation. Inzwischen wird der Schweizer Verein unter dem Namen „Gemeinsam gegen Landminen" weitergeführt ${ }^{44}$.

\subsection{Chemiewaffenabrüstung}

Im März 2003 hat der Ständerat als Zweitrat den Rahmenkredit von 17 Millionen Franken über eine Dauer von fünf Jahren für die Umsetzung des Chemiewaffenübereinkommens (CWÜ) gutgeheissen. Mit dem Geld sollen mehrheitlich Projekte zur Vernichtung von Chemiewaffen in Russland finanziert werden.

미 Jahrbuch 2003, Nr. 1, Kap. 11.7., „Chemiewaffenabrüstung“, S. 215-216.

$41 \quad$ Pressemitteilung Amnesty International Sektion Schweiz, Bern, 9. Oktober 2003.

42 Der Landmine Monitor Report 2003 der International Campaign to Ban Landmines berichtet über die Fortschritte und Rückschläge im Kampf für eine Welt ohne Personenminen, <www.icbl.org/lm/>.

43 Landmine Monitor Report 2003, <www.icbl.org/lm>.

44 Tagesanzeiger, 28. Juni 2003. 
Ein von Green Cross und dem EDA veranstaltetes Genfer Forum für die Vernichtung von Chemiewaffen widmete sich im Juni 2003 dem Thema der Eliminierung der russischen Chemiewaffenbestände. Ziel der Veranstaltung war es, die Abrüstungsbemühungen effizienter zu gestalten und die immer grösser werdende Gruppe international beteiligter Partner besser zu koordinieren ${ }^{45}$. Dadurch soll das Risiko teurer Fehlinvestitionen verringert werden.

Anlässlich der Konferenz erschien das Faltblatt „Chemiewaffenabrüstung Swiss Update" des EDA. Dieses informiert über das Chemiewaffenübereinkommen und den Abrüstungsbeitrag der Schweiz ${ }^{46}$.

Ausserdem hat der Bund beschlossen, an der ,Globalen Partnerschaft gegen die Weiterverbreitung von Massenvernichtungswaffen“" teilzunehmen ${ }^{47}$. Die von den G-8-Staaten gegründete Partnerschaft soll Terroristen und denjenigen, die ihnen Unterschlupf gewähren, am Zugang zu Massenvernichtungswaffen hindern. Bis zu 20 Milliarden US-Dollar werden in erster Linie Projekten in Russland zugute kommen (Chemiewaffenabrüstung, Entsorgung ausrangierter Nuklear-U-Boote, zivile Beschäftigung von Wissenschaftlern, welche im Bereich der Massenvernichtungswaffen gearbeitet hatten).

Die Partnerschaft bringt der Schweiz bessere Koordinationsmöglichkeiten und günstigere Rahmenbedingungen für ihre Projekte, ohne dass sie weitere finanzielle Verpflichtungen eingehen muss.

\subsection{Armee XXI: Friedens- und sicherheitspolitische Elemente}

Nach der klaren Zustimmung von Volk und Ständen zur Änderung des Bundesgesetzes über die Armee und die Militärverwaltung sowie zur Änderung des Bundesgesetzes über den Bevölkerungsschutz und den Zivilschutz am 18. Mai 2003 kann der Bund das Projekt Armee XXI realisieren und den Zivilschutz reformieren. Die neue Armee, welche sich an die veränderte sicherheitspolitische Lage anpasst ${ }^{48}$, wird kleiner und kostengünstiger sein; ihre Flexibilität, Multifunktionalität und Interoperabilität werden erhöht ${ }^{49}$.

Jahrbuch 2002, S. 215-216; 2001, S. 284-285; 2000, S. 226-227.

Da die meisten Gefahren und Bedrohungen grenzüberschreitenden Charakter besitzen, verstärkt die Armee ihre internationale Zusammenarbeit in der Ausbildung, der Rüstungsbeschaffung und in friedensunterstützenden Einsätzen ${ }^{50}$. Die Schweiz arbeitet in diesen Bereichen vor allem mit den Nachbarländern sowie mit Schweden, Finnland, Grossbritannien und den USA zusammen ${ }^{51}$. Da die

45 Green Cross Schweiz Magazin 02/2003; Chemiewaffenabrüstung Swiss Upate, EDA, Juli 2003.

46 Pressemitteilung EDA, 25. Juni 2003.

47 Pressemitteilung EDA, 28. Mai 2003.

48 Als Grundlage dienen der Bericht der Kommission Brunner (26. Februar 1998) und der Bericht des Bundesrates über die Sicherheitspolitik der Schweiz (SIPOL B 2000, 7. Juni 1999).

49 VBS, Dossier Abstimmung 18. Mai 2003 und Bericht des Bundesrates über die Konzeption der Armee XXI (Armeeleitbild XXI, 24. Oktober 2001) (BB1 2002 967).

50 Auch die Zusammenarbeit unter Nachrichtendiensten sowie in- und ausländischen Partnern soll verstärkt werden, Pressemitteilung VBS, 26. September 2003.

51 Im Bereich der Zusammenarbeit hat die Schweiz im September 2003 mit Frankreich und Deutschland verschiedene Vereinbarungen getroffen, Pressemitteilungen VBS, September 2003. 
meisten dieser Staaten dem nordatlantischen Verteidigungsbündnis angehören, ist die Zusammenarbeit auch auf die NATO ausgerichtet.

Mit der Armee XXI möchte sich der Bund stärker an internationalen friedensunterstützenden Operationen und humanitären Hilfeleistungen beteiligen ${ }^{52}$. Der Einsatz militärischer Mittel wird sich auf die Bereiche Schutz, Logistik, Kommunikation und Rettung beschränken. Bei der Unterstützung humanitärer Hilfeleistungen werden die Mittel der Armee einer zivilen Organisation zur Zusammenarbeit zugewiesen. Auch nach der Reform bleibt der zentrale Auftrag der Armee die Verteidigung der Schweiz gegen militärische Bedrohungen.

\section{QUELLEN}

Bund, Swiss Peace Supporter, Journal der Schweizer Beiträge zur internationalen Friedensförderung, Bern, Ausgaben 2003.

EDA, POLITORBIS, das Magazin des Zentrums für Analyse und prospektive Studien, 1/2003 Federalism, Bern, 2003.

EDA, Jahreskonferenz der Politischen Abteilung IV, Menschliche Sicherheit - Sri Lanka, Schlussdokument, Bern, 2003.

Amnesty International and Oxfam, International Shattered Lives, the case for tough international arms control, $2003:<$ www.controlarms.org $>$.

Graduate Institute of International Studies, Small Arms Survey 2003 - Development Denied, Genf, 2003 : <www.smallarmssurvey.org>.

Human Security Network News Bulletin, Volume 2, Issue 1-3: <www.hsph.harvard.edu/hpcr/human security.htm>.

Human Rights Watch, Landmine Monitor Report 2003 : Toward a Mine-Free World, 2003 : <www. icbl.org/lm>.

Kompetenzzentrum Friedensförderung, KOFF Newsletter, Jahrgang 2003, Bern, 2003.

Peace Brigades International, Rundbrief 01/03, Hamburg, 2003.

Ribaux, Claude André, Friedensförderung und Konfliktbearbeitung in der Schweiz. Eine Bestandesaufnahme. Typotron, St. Gallen, 2003.

Schweizerische Stiftung für Minenräumung, Jahresbericht 2002, Genf, 2003.

Trachsler Daniel, „Menschliche Sicherheit: Konzept und Praxis“, in Wenger Andreas (Hg.), Bulletin 2003 zur schweizerischen Sicherheitspolitik, Forschungsstelle für Sicherheitspolitik der ETHZ, Zürich, 2003.

Dahinden Martin, „Die Schweiz und die Ächtung der Personenminen“, in Wenger Andreas (Hg.), Bulletin 2003 zur schweizerischen Sicherheitspolitik, Forschungsstelle für Sicherheitspolitik der ETHZ, Zürich, 2003.

52 In diesem Zusammenhang haben sich Nationalrat und Ständerat mit grosser Mehrheit für die Weiterführung des Swisscoy-Einsatzes im Kosovo bis Ende 2005 ausgesprochen (Geschäft des Bundesrates 03.024). 


\section{INTERNET-ADRESSEN}

Amnesty International Sektion Schweiz: <www.amnesty.ch>.

Berghof Forschungszentrum für konstruktive Konfliktbearbeitung : <www.berghof-Centre.org $>$.

Centre for Humanitarian Dialogue: $<w w w . h d c e n t r e . o r g>$.

Die Bundesversammlung - Das Schweizer Parlament: <www.parlament.ch $>$.

E-Mine: The Electronic Mine Information Network: <www.mineaction.org $>$.

Geneva Call: <www.genevacall.org/home.htm>.

Green Cross Schweiz: <www.greencross.ch>.

International Action Network on Small Arms : <www.iansa.org $>$.

Internationale Kampagne für das Verbot von Landminen (ICBL) : <www.icbl.org $>$.

Kampagne für kontrollierten Waffenhandel: <www.controlarms.org $>$.

Netzwerk Menschliche Sicherheit : <http://humansecuritynetwork.org >.

Oxfam Deutschland: <www.oxfam.de $>$.

Peace Brigades International : <www.peacebrigades.org $>$.

Schweizerische Kampagne gegen Personenminen: <www.uxo.ch>.

Swisspeace : <www.swisspeace.org > 